\title{
Status Report on the LHC Main Magnet Production
}

\author{
G. de Rijk, M. Bajko, M. Cornelis, M. Durante, P. Fessia, J. Miles, M. Modena, G. Molinari, J. Rinn, F. Savary, \\ K. Schirm, F. Simon, D. Tommasini, T. Tortschanoff, and J. Vlogaert
}

\begin{abstract}
The LHC ring will contain 1232 main dipole and 382 main quadrupole double aperture magnets. All main magnets are superconducting and employ $\mathrm{Nb}$ - $\mathrm{Ti} / \mathrm{Cu}$ Rutherford type cables operated at $1.9 \mathrm{~K}$. The dipole production has reached the equivalent of almost three octants of cold masses and nearly four octants of collared coils. The quadrupole production has reached 75 cold masses and over 150 bare magnets. The ramping up of large scale magnet production has posed several challenges which will be discussed, like: the coil size uniformity, coil pre-stress control, cold mass welding technique and the geometrical shape issues. The magnetic measurement results at warm will be presented together with their usage for the quality control in the production. The common features and differences of the three dipole producers will be discussed. The latest version of the production schedule will be presented.
\end{abstract}

Index Terms-Production control, superconducting accelerator magnets.

\section{DipOLE INTRODUCTION}

$\mathbf{T}$ HE production of the LHC dipoles is carried out at three firms (Cold Mass Assemblers) and the contracts have been divided in a contract of 30 pre-series Cold Masses for each CMA and a contract of 386 series CMs for each CMA. At present all three CMAs are well into the series contract (see Fig. 1). BNN (D) produced $201 \mathrm{CMs}$ and will probably finish the production by end 2005. The Alstom-Jeumont consortium (F) produced $126 \mathrm{CMs}$ and Ansaldo Superconduttori (I) produced $87 \mathrm{CMs}$. The latter two are planned to complete their production by summer 2006 .

\section{The Production Evolution FOR THE DiPOLES}

\section{A. Cross Section Evolution}

Fig. 2 shows the cross section of the dipole CM. The crosssection of the coil has slightly changed during the pre-series and series production. After 35 cold masses, in order to correct for the most important multipole components $\left(\mathrm{b}_{3}, \mathrm{~b}_{5}\right.$ and $\left.\mathrm{b}_{7}\right)$ the dimensions of the copper wedges in the coil inner layer were slightly modified to fine tune the field. After another 146 cold masses a $0.125 \mathrm{~mm}$ polyimide shim was added to the coil mid plane to further correct the field quality.

\section{B. Production Rate}

BNN is producing at a rate of up to $20 \mathrm{CMs}$ per month and will end the production mid 2005. Alstom-Jeumont has

Manuscript received October 3, 2004.

G. de Rijk, M. Bajko, M. Cornelis, P. Fessia, J. Miles, M. Modena, G. Molinari, J. Rinn, F. Savary, K. Schirm, D. Tommasini, T. Tortschanoff, and J. Vlogaert are with the Accelerator Technology Department, CERN, 1211 Geneva 23, Switzerland (e-mail: Gijs.de.Rijk@cern.ch).

M. Durante and F. Simon are with CEA, Saclay, France.

Digital Object Identifier 10.1109/TASC.2005.849500

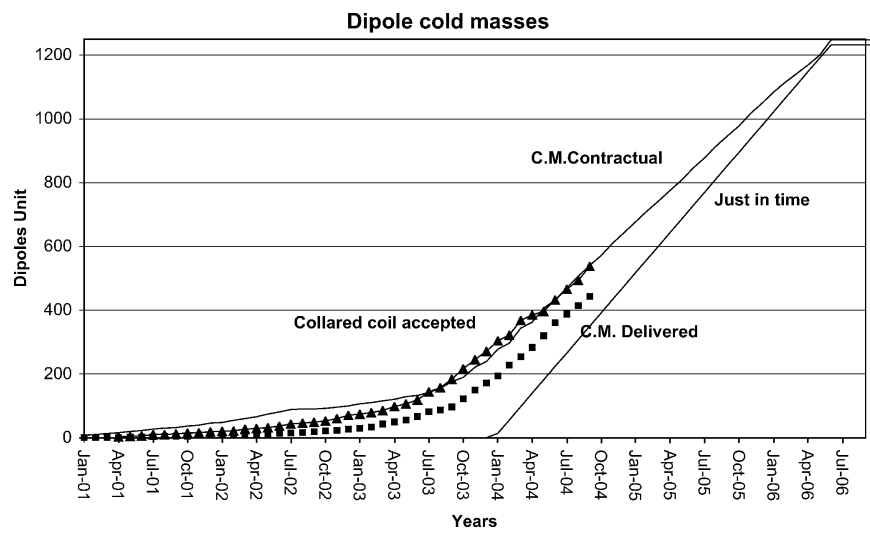

Fig. 1. Cold mass deliveries of the 3 cold mass assemblers (from the LHC dashboard [1]).

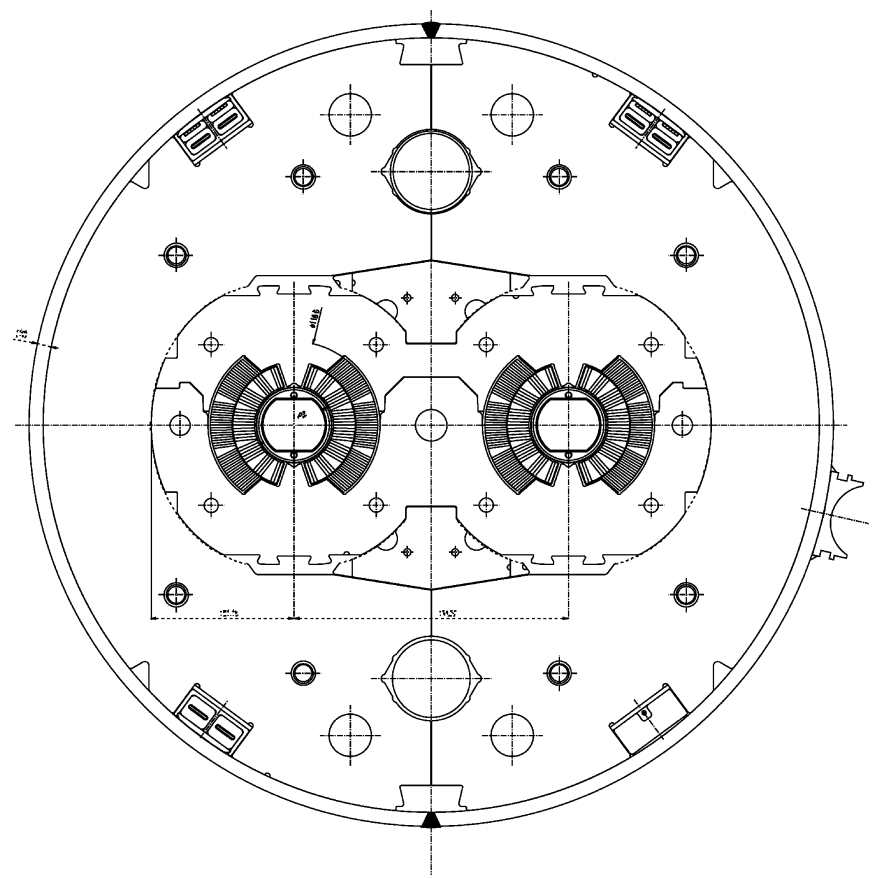

Fig. 2. The LHC dipole, cross section of the cold mass.

achieved a rate of $12 \mathrm{CMs}$ per month taking them into summer 2006 to complete the contract. Ansaldo is still ramping up the production rate but is also planning to end in summer 2006.

\section{Production Technique}

The dipoles, which are made by the 3 CMAs, are in principle identical. However, some small differences were allowed. Detailed descriptions of the project are given in [2]-[5].

1) Coil End Shape: The coil end spacers of BNN and Ansaldo are basically as designed by the CERN design 


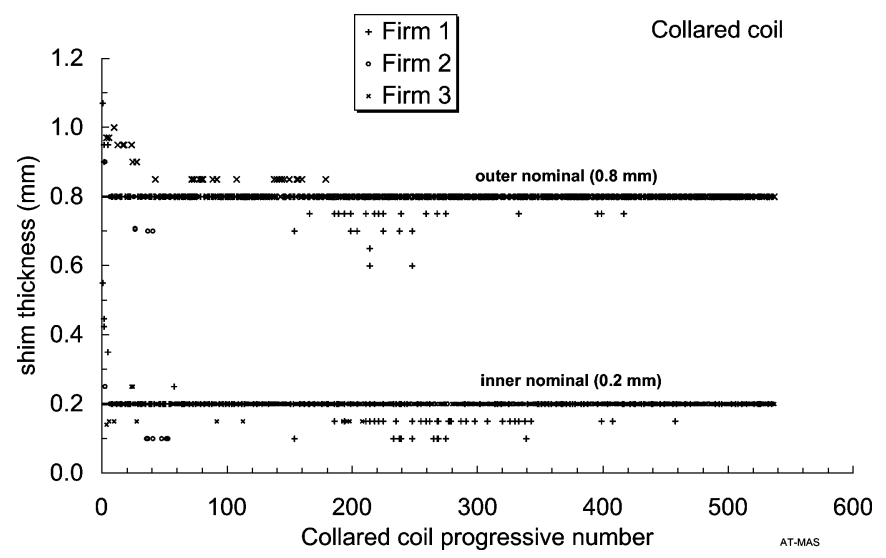

Fig. 3. The collar-to-coil shims for the first 540 dipole collared coils.

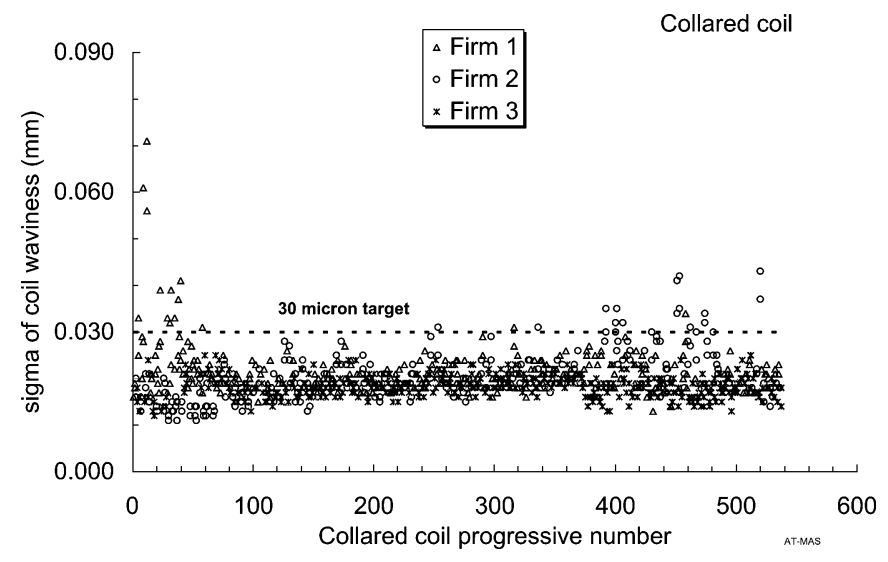

Fig. 4. Coil waviness for the first 540 dipole collared coils. It is defined as the standard deviation of the movement of all coil blocks needed to give a multipole variation in agreement with the magnetic measurements. Magnetic measurements are done at warm on the dipole collared coils.

team (recently BNN has slightly modified one spacer) while Alstom-Jeumont preferred a slightly modified set of spacers for the whole production. All three versions have shown equivalent quench performance.

2) Coil Size and Press Stress: In order to guarantee the coil pre-stress over the whole $15 \mathrm{~m}$ length, the coil size, measured under pressure, should remain within tight tolerances. At a given collar cavity the tolerance of the pre-stress is $\pm 15 \mathrm{MPa}$. This corresponds to a size variation of $0.12 \mathrm{~mm}$. For this purpose, coil sizes are measured under a press at the nominal pressure of $100 \mathrm{MPa}$. From this measurement the dimensions are extrapolated at the working pre-stress of 70-80 MPa.

If a coil is outside the target window, the global coil size can be adjusted varying the existing shims at the pole faces of the inner and outer layers. In Fig. 3 a plot of the pole shims for the collared coils manufactured so far can be found [6]. Fig. 4 shows a measure of the homogeneity of the coil size along the $15 \mathrm{~m}$ length defined as "waviness". The problems of one CMA at the beginning of the production were mastered by improvements to the curing tooling and procedures, but a tight quality control of the coil production is needed to keep the waviness low.

3) Collaring: The coils are wrapped in four layers of $0.125 \mathrm{~mm}$ thick ground insulation (Apical) and surrounded by two layers of coil protection sheets each $0.5 \mathrm{~mm}$ thick. Ansaldo uses stainless steel protection sheets (YUS $130 \mathrm{~S}$ ), BNN uses stainless steel on the coil ends and a special alloy $\left(\mathrm{Cu}_{61} \mathrm{Ni}_{18} \mathrm{Zn}_{21}\right)$ on the straight parts while Alstom-Jeumont uses a bronze alloy over the full length. The collar laminations are preassembled in packs with pins, before the collaring process. The collar packs are of different length at the three CMAs and in the case of Alstom-Jeumont the pack is just a collar pair. The upper and lower collar halves are locked together by three rods. All three solutions have shown to be equivalent in terms of magnetic performance.

4) Cold Mass Welding: The cold mass is surrounded by a $10 \mathrm{~mm}$ thick $316 \mathrm{LN}$ stainless steel shrinking cylinder. The welding of the half-shells forming the cylinder is done in a welding press, where both welding seams are made at the same time by two semi-automatic welding torches traveling along the press. The weld consists of four passes, one Surface Tension Transfer pass and three Metal Active Gas filling passes. The resulting pre-stress in the shrinking cylinder is $\sim 150 \mathrm{MPa}$ where $120 \mathrm{MPa}$ is due to the weld shrinking (mainly STT pass) and $30 \mathrm{MPa}$ due to the applied pressure of $\sim 500 \mathrm{t} / \mathrm{m}$. The commissioning of the welding presses and the industrialization of the entire process took longer than expected as new and automated welding technologies were applied. The three CMAs mastered the technique only after several tens of CMs of the series production. The problems encountered were: lack of fusion in the welds, welding porosities, too long welding times and CM shape errors. To solve the problems, CERN formed a task force who together with the CMAs upgraded the various control and detection systems, improved the welding parameters and provided more in depth training for the welding press operators.

\section{Dipole Quality CONTROL}

The production of a large series of magnets, which are near to the limit of today's technology, requires an important effort of quality control. During the production the following measurements are made:

1. Coil size samples on straight part and coil ends to determine the shims.

2. Electrical integrity of all layers, poles, collared coils and cold masses.

3. Magnetic field with rotating coils, at warm, for all collared coils and all finished cold masses.

4. Collared coil transverse size.

5. Geometrical shape of the cold mass, after welding and after completion.

6. Weld quality by X-rays.

7. Leak tightness at 25 bar He pressure.

The data from all the measurements (and other production data) are stored at CERN in a database (Manufacturing and Test Files). At fixed points in the production there are so called 'holding points'. At a holding point, a set of data is analyzed at CERN and a message is issued by the analysis team: Ok, Warning or Hold. On the basis of this, the Project Engineers can decide to continue the assembly or to demand a rework. Holding points are: 


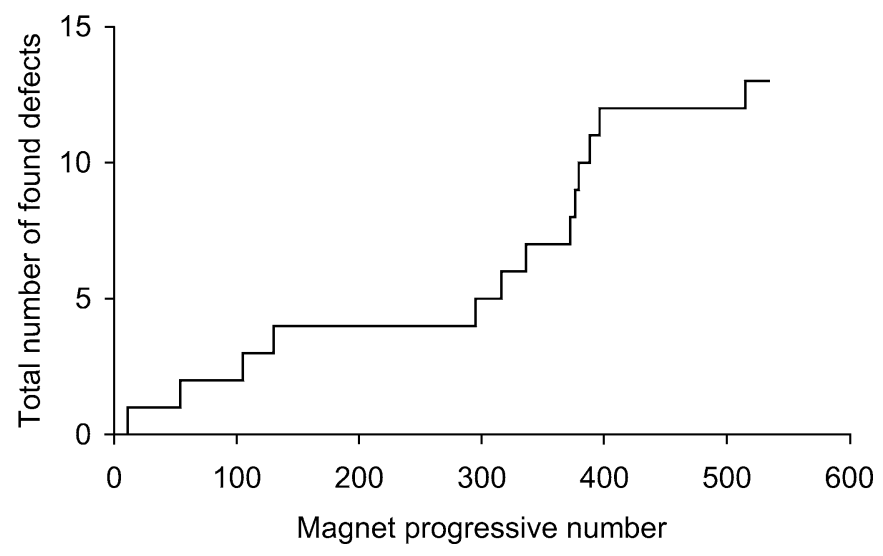

Fig. 5. Defects found on collared coils with the warm magnetic measurements.

1. Coil shimming (collar-to-coil) proposal of the straight part shims before collaring based on the coil size measurements.

2. Collared coil warm magnetic measurements.

3. Cold mass shape.

4. Cold mass warm magnetic measurements.

The holding point on the shimming guarantees a control of the shim size evolution. A nonnominal collar-to-coil shim size (see Fig. 3) implies unwanted magnetic field errors and should therefore be minimized. The holding point on the warm magnetic measurements on the collared coils allows the detection of assembly and fabrication errors generating displacement of the conductors. The measurements are done with a $75 \mathrm{~cm}$ long 'mole' equipped with rotating coils. This measurement results in a full multipole knowledge in 20 longitudinal positions. Anomalies in the multipoles give rise to the warning or hold actions. With the help of a magnetic computation model a prediction can be made of the type and magnitude of the errors. To gain longitudinal precision in the location of the anomaly, before opening a collared coil, measurements can be repeated with a shorter $(15 \mathrm{~cm})$ mole. On the basis of these predictions a decision can be taken to open a collared coil.

In Fig. 5 a plot of the number of defects detected with this method can be found [6]. During the series production (Fig. 5 magnets 300-400) such cases occurred independently at all three CMAs.

In one company the reason for most of the displaced windings was poor gluing of the coils. In all three CMAs poor control of the curing cycle for the coil gluing, due to different reasons, was the cause of many problems. These incidents prompted a tighter surveillance of the curing cycles at all three CMAs. In one company the displacements seem to have their origin in the collaring rather than in the curing and the analysis to understand this is under way.

The holding point of the magnetic measurement of the cold mass has not revealed any anomalies up to now. It is however important to determine the effective magnetic length and to obtain a cross-check of the collared coil data.

The holding point on the cold mass shape allows the control of the evolution of the shape [7]. The CM is curved in the horizontal plane and has a nominal sagitta of $9.14 \mathrm{~mm}$ with a toler-

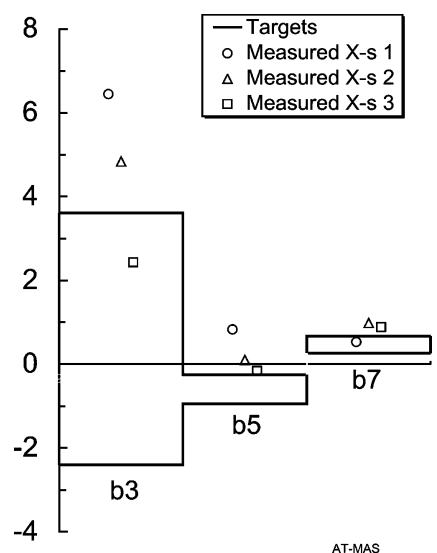

Fig. 6. The average values of the lower order systematic odd normal multipoles (see Section II-A for the cross sections 1, 2 and 3).

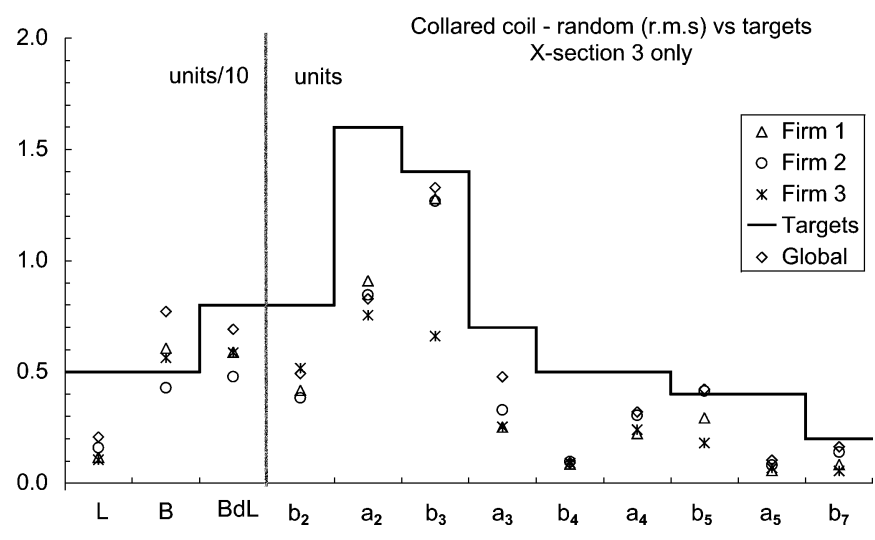

Fig. 7. The random field components as obtained for the three CMAs.

ance of $\pm 1.5 \mathrm{~mm}$. The final sagitta is the result of many parameters; e.g. status of the half shells (shape and residual stress), press geometry and internal friction inside the cold mass. When this amount of bending was found to drift outside the tolerance during production, a modification of the imposed bend on the welding press was introduced. The twist of the CM (horizontal angle over the $15 \mathrm{~m}$ length) is closely monitored. A problem with the twist at one CMA was solved by a re-alignment of the welding press.

The warm magnetic measurements of the collared coils are also used to follow the field quality and to assure that the multipole parameters stay within the limits defined by the beam dynamics studies. The two modifications on the coil cross section mentioned in Section II-A were originating from these studies [10]. In Fig. 6 the average odd normal multipoles $b_{3}, b_{5}$ and $\mathrm{b}_{7}$ measured for the three cross sections are shown. In Fig. 7 the random field components can be found. The electrical tests are not subject to a holding point but resistance values, ground insulation resistance values and discharge reaction are required to stay within well defined limits. Nevertheless, the most delicate construction aspect is the electrical integrity of the CM. A small number of magnets showed electrical problems at cold. Three CMs were completely dismounted and repaired due to electrical faults (e.g. quench heater connection and insulation, inter-turn short and cold welds in the superconducting cable). 


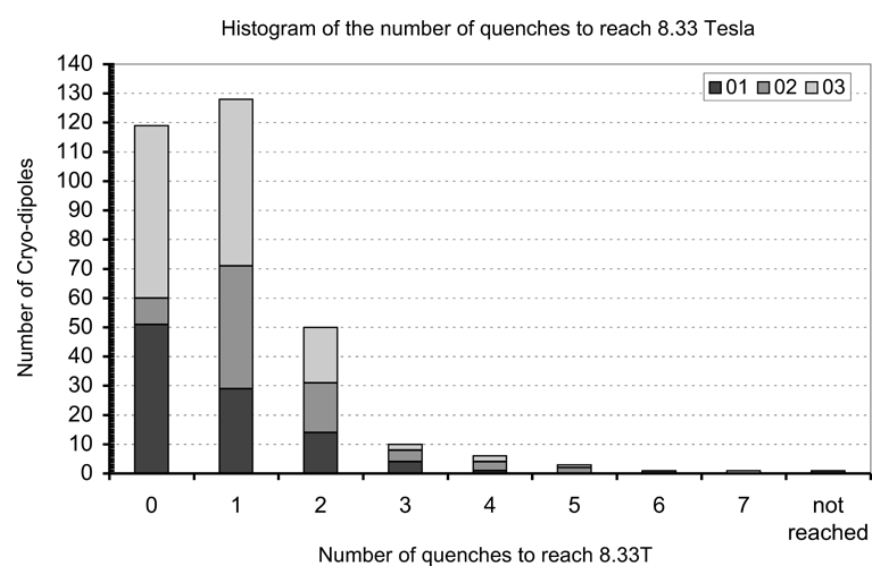

Fig. 8. Histogram showing the number of quenches per CM to reach the nominal field of $8.33 \mathrm{~T}$ at $11850 \mathrm{~A}$ at the first thermal cycle.

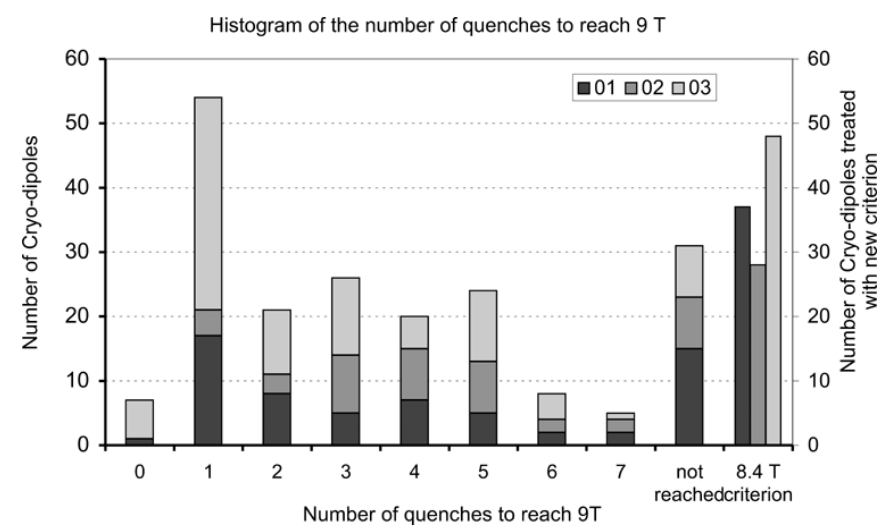

Fig. 9. Histogram showing the number of quenches per $\mathrm{CM}$ to reach the nominal field of $9 \mathrm{~T}$ at $12850 \mathrm{~A}$ at the first thermal cycle.

\section{Dipole Cold Test Performance}

Each CM is tested at CERN and is subjected to at least one cool-down and two "performance" quenches. The tests consist of: vacuum tests, electrical integrity tests before cool-down, at cold (before and after training) and again after warm-up. Up to the beginning of 2004 the quench training was done up to $9 \mathrm{~T}$ for all CMs.

In the beginning of 2004 a backlog of up to $120 \mathrm{CMs}$ out of $200 \mathrm{CMs}$ delivered, waiting for cold tests, had accumulated. In order to resolve this backlog magnetic measurements at cold were reduced from $100 \%$ down to $30 \%$ and a reduced quench training procedure was defined.

$\mathrm{CMs}$, which reach $8.4 \mathrm{~T}$ during the first two quenches $(8.33 \mathrm{~T}$ being the operational value), are not further trained. The CMs, which do not fulfill this criterion, are trained toward the ultimate field of $9 \mathrm{~T}$. This procedure was based on the experience of the pre-series CMs where all the CMs reaching $8.4 \mathrm{~T}$ in two quenches also reached the ultimate field in the specified nine current ramps. CMs which display a quench training outside specification are subject to technical and commercial re-evaluation. Fig. 8 shows a histogram with the number of quenches needed to reach the nominal field of $8.33 \mathrm{~T}$ during the first thermal cycle. Fig. 9 shows the number of quenches necessary to reach the ultimate field of $9 \mathrm{~T}$, at the first thermal cycle, for the CM where this was attempted. The majority of quenches
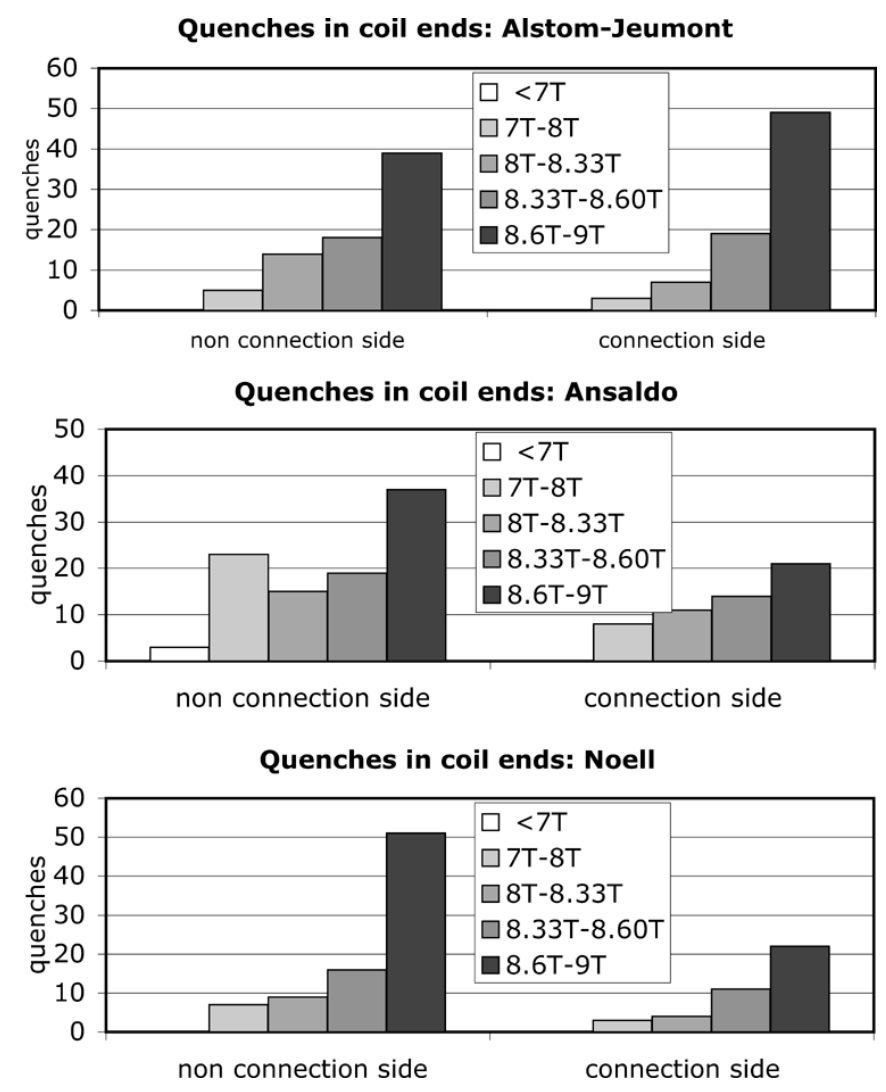

Fig. 10. Distribution of quenches in the dipole coil ends between connection and nonconnection site of the $\mathrm{CM}$ as function of the field values when they occurred.

( $>90 \%$ ) occurs in the coil ends. In Fig. 10 the distribution of quenches at the two ends can be found. The distributions are different for the three CMAs. Investigations are ongoing to try to understand this.

\section{QUADRUPOLE PRODUCTION EVOLUTION}

\section{A. Production Progress}

The LHC main quadrupole project is a collaboration between CERN and CEA-Saclay (France). The magnet design was done by CEA, CERN being responsible for the contract, the components and interface problems and both partners do the project follow-up together. The quadrupole series production is well under way at ACCEL Instruments (Germany) [8]. In total $360 \mathrm{CMs}$ were ordered for the main Short Straight Sections, $32 \mathrm{CMs}$ for the Dispersion Suppressor sections and 8 spare CMs were ordered as not assembled units. More than half of all the coils and more than 150 bare quadrupoles have been produced. By September 200475 SSS CMs have been delivered to CERN. The coil production and the single aperture assembly (the design is based on separate apertures) are in advance of the CM finishing. In Fig. 11 the production progress is shown. Each SSS CM contains a quadrupole, a sextupole-dipole corrector on one end and an other corrector magnet, either an octupole, tuning quadrupole or skew quadrupole, on the opposite end. There are 40 variants of the SSSs, each with different corrector combinations, protection diode polarities and cryostat interfaces. 

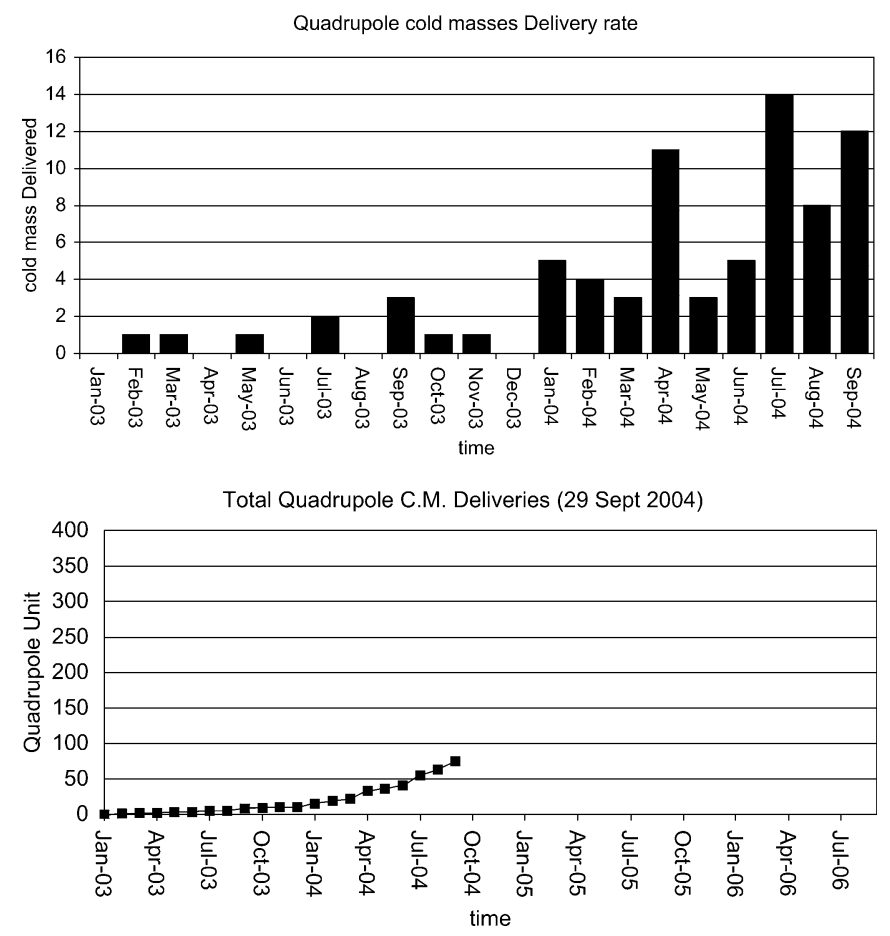

Fig. 11. Delivery rate and total deliveries of quadrupole CMs.(SSS) [4].

The quadrupoles are manufactured at ACCEL but the other corrector magnets as well as numerous other components are made by other suppliers and are delivered to ACCEL by CERN. ACCEL assembles the magnets into the $6 \mathrm{~m}$ long SSS and Dispersion Suppressor CMs. The assembly comprises: fitting: all the magnets into an inertia tube, the routing of all the bus bar connection, fitting cold bore tubes, heat exchanger tube, filling pieces, diodes and the instrumentation. The SSS CM cold tests are done at CERN and are reported in a separate presentation [9]. The production of the SSS, with corrector magnets and other components coming from many suppliers, poses regularly supply challenges. At the moment the supply problems of corrector magnets have been solved but the supply in time of the superconducting bus-bars has no margin.

\section{B. Coils}

In Fig. 12 a cross section of the main LHC quadrupole can be found. The cross section remained unchanged apart from a correction in the coil to improve the $\mathrm{b}_{6}$ component at injection current with a modified thickness of the coil protection sheets (starting at aperture 50) and a modified mid plane shim (starting from aperture 150). In Fig. 13 a plot of the thickness of the coil protection sheets used in the quadrupoles is reported [11].

The homogeneity of the coil size over the length of the coils can be seen in Fig. 14, where the coil waviness is shown for individual apertures (two apertures per CM) as deduced from the warm magnetic measurements made on the collared coils. While in the beginning this was within target, the waviness has increased for the second part of the production.

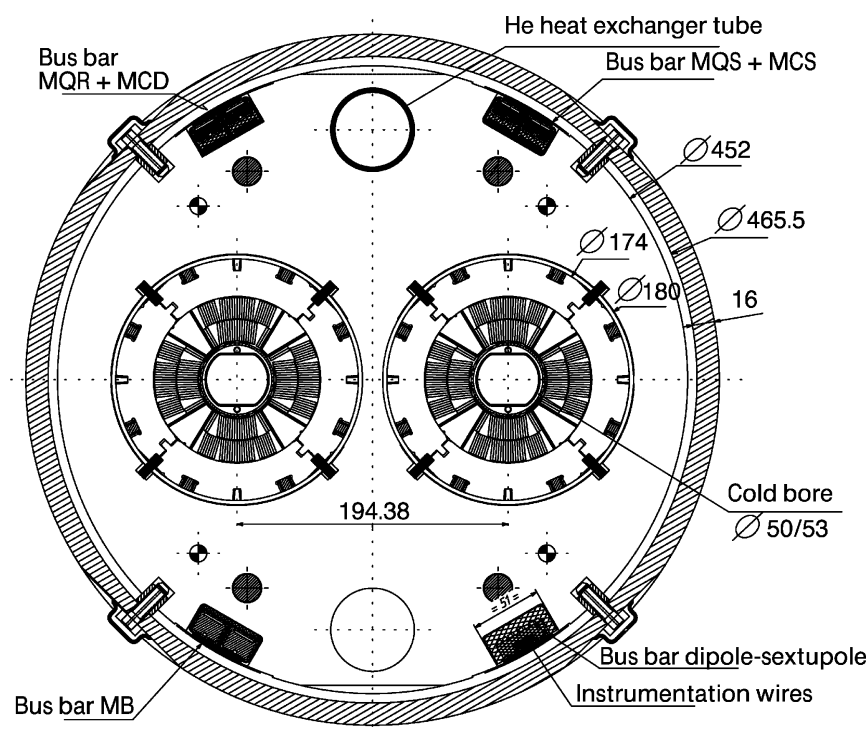

Fig. 12. The LHC quadrupole cross section.

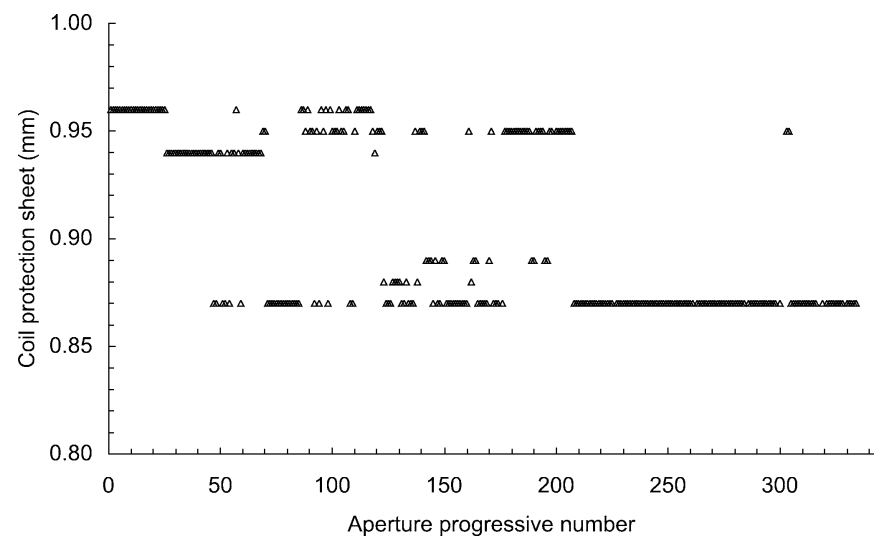

Fig. 13. The coil protection sheets employed in the quadrupoles.

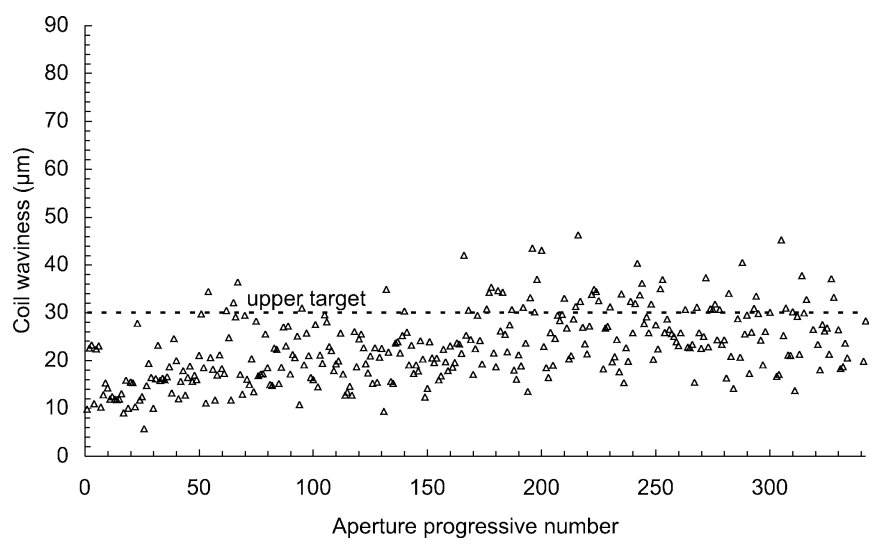

Fig. 14. Quadrupole coil waviness as inferred from the warm magnetic measurements on the collared coils.

\section{QUADRUPOLE QUALITY CONTROL}

The quality control of the SSS production in done in the same way as for the dipoles, and the data are stored in the same database (MTF). Holding points are used to control the electrical, 

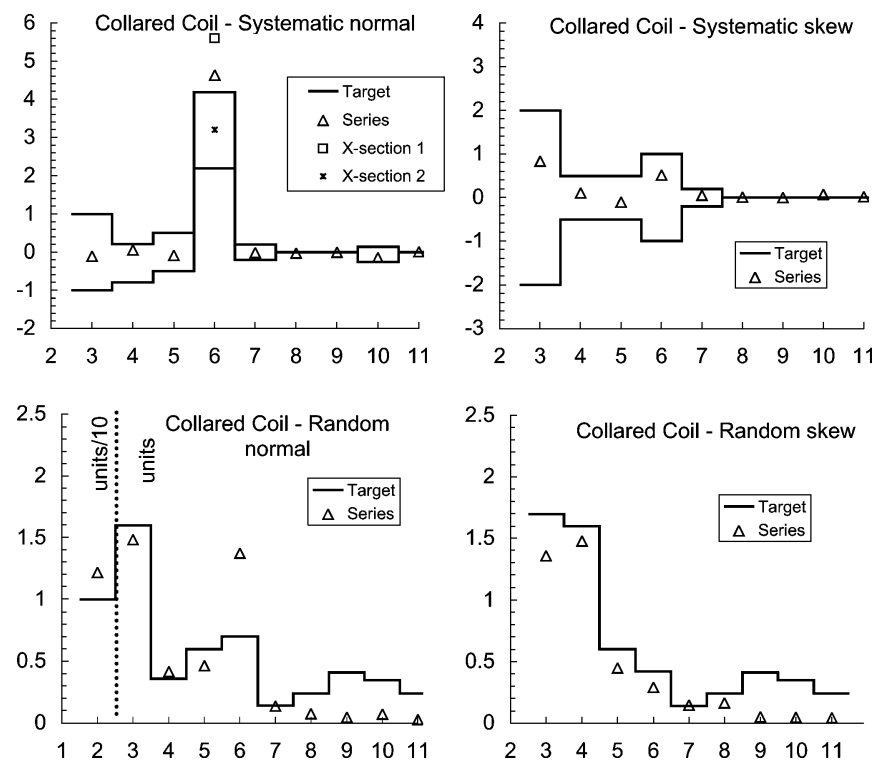

Fig. 15. Systematic and random multipoles for the quadrupoles as measured on the collared coils at warm.

magnetic and geometrical parameters of the coil, collared coil and CM.

A more critical aspect of the SSS production is the many types of corrector magnets and the resulting connections. The superconducting bus bar geometry is complex and special care is needed to assure the correct magnet polarity in the 40 variants of the cold masses. During the beginning of the production problems occurred with the polarities of the corrector magnets. These problems were traced down to labeling insufficiencies.

Also for the quadrupoles the warm magnetic measurements of the collared coils are used to follow the field quality and to assure that the field quality stays within the limits defined by the beam dynamics studies. Fig. 15 shows the systematic and random field components.

\section{CONCLUSION}

The production of the LHC dipoles and quadrupoles is well under way with almost three octants worth of magnets delivered.
Nevertheless the production is very sensitive to small errors and a tight quality control and a short reaction time on problems are crucial for the success. Both the dipoles and quadrupole production are keeping the schedule. The dipoles are foreseen for completion in summer 2006 and the quadrupoles by spring 2006.

\section{ACKNOWLEDGMENT}

The effort for the dipoles and the quadrupoles is the work of a large number of people in many institutes and firms. The authors would like to thank all the members of the AT/MAS group for their contribution to this paper. The authors would like to thank C. Vollinger, P. Hagen and E. Wildner for the their work on the holding points and E. Todesco for providing the statistics. In particular they would like to thank L. Rossi and L. Evans for their lasting support and encouragement on these magnet projects.

\section{REFERENCES}

[1] LHC Dashboard. [Online]. Available: http://lhc-new-homepage.web. cern.ch/lhc-new-homepage/DashBoard/index.asp

[2] "LHC Design Report," CERN, 2004-003, Jun. 4, 2004.

[3] "Technical Specification for the Supply of 1158 Cold Masses of the Superconducting Dipole Magnets for the LHC Collider,", Geneva, LHC-MB-CI-0006, LHC-MMS/98-198 Rev. 2.0, May 2001.

[4] L. Evans, "Status of the Large Hadron Collider (LHC)," IEEE Trans. Appl. Superconduct., vol. 14, no. 2, p. 147, Jun. 2004

[5] L. Rossi, "Superconducting magnets for the LHC main lattice," IEEE Trans. Appl. Superconduct., vol. 14, no. 2, p. 153, Jun. 2004.

[6] E. Todesco. Report on Field Quality in the Main LHC Dipole Collared Coils and Cold Masses: June-July 2004. [Online]. Available: http://lhcdiv-mms.web.cern.ch/lhc-div-mms/MMSPAGES/MA/Obs.html

[7] E. Wildner et al., "The Geometry of the LHC Main Dipole,", Geneva, LHC-Project-Report-729, July 2004.

[8] R. Burgmer et al., "Performance of the first LHC main quadrupoles made in industry," in Proc. PAC2003 Conf., Portland, OR, USA, May 12-16, 2003.

[9] S. Sanfilippo, "Field quality and quench performance of the first LHC short straight sections," IEEE Trans. Appl. Superconduct., submitted for publication.

[10] E. Todesco et al., "Steering field quality in the main dipole magnets of the large hadron collider," IEEE Trans. Appl. Superconduct., vol. 14, no. 2, p. 177, Jun. 2004.

[11] E. Todesco. Report on Field Quality in the Main LHC Quadrupole Collared Coils and Cold Masses: July-August 2004. [Online]. Available: http://hc-div-mms.web.cern.ch/lhc-div-mms/MMSPAGES/MA/ qobs.html 